\title{
Aircraft Fuselage Recent Developments - A Review
}

\author{
Sohan Angelo ${ }^{1}$, Varun Potty ${ }^{1}$, P. Srinivasa Rao ${ }^{2}$, Srinivas $\mathbf{G}^{3,{ }^{*}}$ \\ ${ }^{1}$ Manipal Institute of Technology, Manipal Academy of Higher Education (MAHE), India \\ ${ }^{2}$ Department of Humanities and Management, Manipal Institute of Technology, Manipal Academy of Higher Education (MAHE), India \\ ${ }^{3}$ Department of Aeronautical and Automobile Engineering, Manipal Institute of Technology, Manipal Academy of Higher Education \\ (MAHE), India
}

Received August 29, 2019; Revised December 17, 2019; Accepted December 24, 2019

Copyright@2019 by authors, all rights reserved. Authors agree that this article remains permanently open access under the terms of the Creative Commons Attribution License 4.0 International License

\begin{abstract}
Over multiple iterations spanning many years of research a stable and aerodynamically workable fuselage structure has been zeroed down on. The fuselage being the segment holding the passengers and crew requires an immaculate degree of stability during takeoff, landing and flight. Aerodynamic optimisation presupposes every notion of this 'in flight stability'. The recent interest taken in the field of stability under unforeseen air conditions has led to remarkable developments in the field of aerodynamics. This paper attempts to categorically classify these interests into 3 sections- Theoretical, Experimental and Numerical. Various mathematical models and algorithms have been created to study and test the stability of the fuselage under turbulent conditions caused by weather. Turbulence caused by on flight equipment (propellers etc) and methods for its mitigation have also been mentioned. The chine angle analysis of the fuselage reveals that a sharper angle is more favorable in increasing the lift. The study of asymmetrical vortices and its evolution has enhanced the field of aerodynamic optimization. Unconventional aircraft designs like the BWB are studied and compared against the incumbent structures. Various modeling softwares like CATIA have extensively been used to design these structures. A compilation of these recent developments has been presented to those attempting to intensively analyse and study the field of aerodynamic stability.
\end{abstract}

Keywords Aircraft Fuselage, Analysis, Aviation industry

\section{Introduction}

The race to develop the most commercially successful and renowned passenger aircraft has shifted gears to focus on research topics closely pertaining to the fuselage. The fuselage is the most recognisable component of the aircraft and it refers to the long hollow tube which encloses the cargo and passengers along with the crew of the plane. The fuselage forms the center piece around which the aircraft structure is built. The fuselage structure has had to adapt with the changing times and working specifications, resulting in four major types - Truss structure, Geodesic construction, Monocoque shell, and Semi-monocoque fuselage. The more commonly adapted method of construction utilized is the Semi-monocoque fuselage owing to its ease of reproducibility in the manufacturing process and ease of working with metals. The general structure of the fuselage varies based on application and prerequisites in the working conditions of the aircraft.

Each aircraft is required to adhere to rigorous regulations, reiterating its ability to come out of worst-case scenarios unscathed, which is attributed to the structural integrity of the fuselage. The gains (loss of weight) made by design alterations to the blueprint of the fuselage, by integrating new age composite materials and changing the fundamental structural design, cannot be at the cost of structural integrity of the fuselage, putting the safety of the aircraft in jeopardy. The fuel consumption of the aircraft is inordinately dependent on its aerodynamic performance through the air. Aerodynamics of the aircraft plays a crucial role in efficiently developing lift without exponentially increasing drag. The aerodynamic design is an aspect that is heavily taken into consideration in the initial stages of the design process. Various additions made to the fuselage over the years has handed the pilot greater control over the plane. The ability of the aircraft to follow the computed trajectory through the 3 phases of flight, and the relatively low latency in reaching equilibrium after hitting disturbances in the air is in part due to the stability of the aircraft. Based on the preconditions, the manoeuvrability and versatility of flight actions can be crafted by altering the stability levels of the fuselage specific to the aircraft model.

Data along various parameters, like the global passenger trips and accessibility to air travel in developing countries, 
see an exponential increase over the past decade with this development to continue in years to come, indicating a greater proliferation and demand for air travel. Seeking to capitalise on this opportunity, the bigger players in the field of aeronautics have made a major push towards reducing the cost incurred by the passenger. This in tandem with increasingly stringent environmental standards has set the future trend for fuselage design to accommodate aircrafts that have a smaller environmental footprint along with increased seating capacity and shorter travel times without compromising on comfort and safety. This implies that fuselage will have to withstand forces at supersonic flight, consist of materials made of lighter and yet stronger composite materials and undergo a transformation in the design philosophy. Plans to make the fuselage structures smarter by incorporating health monitoring and fault tolerant mechanism and more robust flight control systems are in the works. Attempts to improve aerodynamic efficiency by means of active flow control systems are also being taken in consideration. In an attempt to improve the optimization process of the fuselage design, three main characteristics are taken into consideration -Structure, Aerodynamics and stability.

\section{Literature Review}

\subsection{Theoretical Analysis of Aircraft Fuselage}

Yii-Mei Huang et al [1]tackles the technique of passive sound management systems. Their main objective was to design dynamic dampening absorbers to eliminate fuselage vibrations generated from external influences like propellers etc. They evaluated the appropriate parameters to be chosen in design process of the absorbers, to optimally keep the vibrations and noise generated it a minimum. They primarily constructed mathematical algorithms for the motion, vibrations, interior sound field and the external forces that affect the cylindrical fuselage. These were further used to determine the optimal design and placement of the absorbers. By considering kinetic energy and potential energy of the fuselage and the sound field as objective functions, they concluded that their approach had a major impact in reducing the undesirable noise in the fuselage structure.

ParthaDey et al [2] understands the stability of composite skew plates when subjected to loads. The dynamic stability of composite skew plates was analyzed using four-noded shear flexible quadrilateral plate. Finite element equations of the plate were formulated. Gaussian integration rule was used to calculate matrices for elemental mass and linear-geometric stiffness. First and second order dynamic instability boundaries were then identified. The identified principal stability region was four times the secondary instable region. As load amplitude increased, the first and the second order approximations differed. Region of instability increased with the increase in skew angle. The paper lays out the influence skew angle, lay-up and static in plane loads have on dynamic stability.

Zhiquan LI et al [3], with previous research on tiltrotors as a basis, intended to construct a full-span model tiltrotor followed by its analysis along the parameter of aeroelastic stability in flight. They also established the differences between a semi and a full span model and nailed down the reasons for its instability and monitored the effects of external structures on its aeroelastic stability. After constructing a theoretical model of the tiltrotor, they constructed algorithms to represent various structures and features of the tiltrotor. These equations were used to validate the various parameters, and the results indicated that elastic blades invariably led to more instability than rigid winged aircrafts did, and when these were coupled with fuselage motions, the instability was found to have increased further.

Natural laminar flows (NLF) wings are capable of maintaining flow under laminar conditions over relatively large portions of wing surface. But imperfections produced during the manufacturing process (groves between joints and fixtures) have an adverse effect on aerodynamic performance due to a disruption in air flow over the wing surface. JesúsGaricano-Mena et al [4] studied the stability characteristics of NLF wings in the presence of these indentations. The main objective was to quantify the impact on linear stability under varying degrees of indentation of depth and Mach number. They first established a baseline test under ideal circumstances, and then observed the modifications made to this data when exposed to a perturbation in the surface of the wing structure. They also noted spatial evolution of individual structural elements along the direction of the flow along the amplification factors. They utilized global temporal stability analysis to make these determinations. The results from the test indicated that an increase in grove depth drastically impacted temporal stability which was intensified at higher Mach numbers. They cautioned that these parameters need to be taken into considerations during the design and manufacturing process of NLF wings.

Liang QU et al [5] studied the stability values for the working parameters (angle of attack and sideslip, yaw, pitch and roll rate) of the aircraft under icing conditions. Apart from increasing the weight of the aircraft, icing also decreases the performance of the aircraft. Stable and unstable equilibrium points for stability of the aircraft were obtained by solving the kinematic equations. The stability region was then obtained by using these equilibrium points in a Jacobi Matrix and solving it. An iced aircraft was in steady state if the angle of attack laid below 25.8 degrees (38.7 degrees for an un-iced vehicle). Time domain simulations carried out validated the stability regions obtained. The stability region provided a working region for the aircraft for safe operation. 
(a)

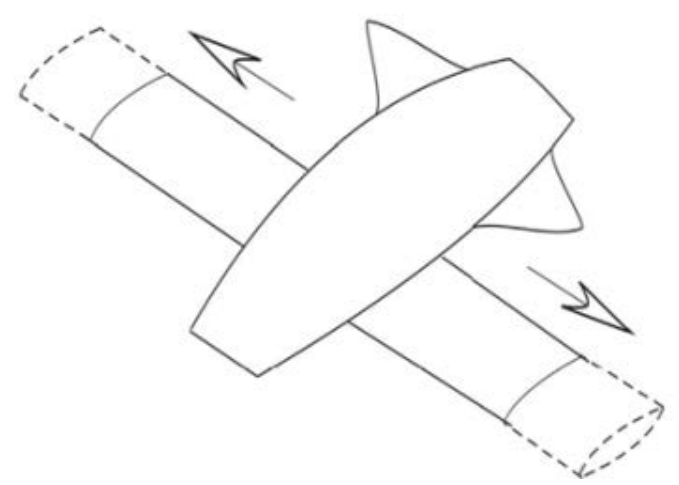

(b)

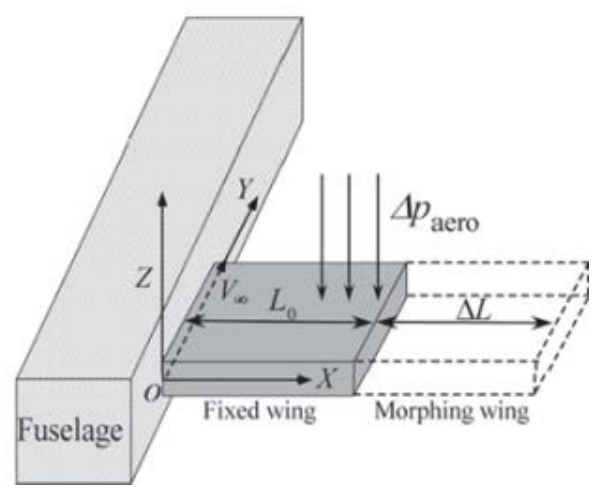

Figure 1. The schematic diagram of morphing aircraft (a) a morphing aircraft with variable-span wings, (b) the simplified model of axially moving wing. [6]

Wencheng $\mathrm{Li}$ et al [6] intended to make headway in morphing aircraft design by devising a strategy to suppress the flutter that occurs beyond a certain span length. The rough schematic of a morphing plane is given in figure 1 . The three main objectives are, to establish equations for an axially moving cantilever kept in supersonic fluid, second, to develop a new strategy for flutter control, and to obtain the boundary conditions for morphing rate by slowing varying the system. After constructing the structural model of the aircraft, it was put through a set of numerical simulations which controlled the rate at which the cantilever plate was extended and retracted, exposing it to varying degrees of aerodynamic loads. They concluded from the results that the speed of the flutter was inversely connected to the span length and it also increased with increase in deploying rates. They suggested that this problem could be overcome by strategically varying the span length along with proper amplitude to improve the quality of flight of the aircraft.

N. Siepenkötter et al [7] considers the nonlinearity of a flexible aircraft and studies its stability. Nonlinear effects on stability need to be considered along with frequency and damping of motion. Eigenvalue analysis incorporates the nonlinear characteristics while modelling motion. The flexible aircraft is modelled using Newton-Euler equations. Forces acting due to air were modelled using strip theory. Stability regions on the system were found beyond the known equilibrium points. The obtained equilibrium values spoke of a trimmed aircraft moving at $100 \mathrm{~m} / \mathrm{s}$. Eigen values obtained were used to identify 9 forms of motion and determine the stability under them. The above modelling can be used to study flexible aircrafts in unsteady aerodynamic conditions as well.

\section{Experimental Analysis of Aircraft Fuselage}

Active noise control systems have the unfortunate effect of amplifying the vibrations in certain regions of the fuselage. C. Natale et al [8] attempted to tackle this problem by conducting an experimental analysis of an active vibration control system on a fuselage panel of BOEING 717 aircraft. The main objective was to construct a mathematical model which is representative of the fuselage, and thereafter to validate the algorithms effectiveness as a tool in the design process experimentally. The experimental setup included a panel along with actuators rated to produce a force of $133 \mathrm{~N}$ and a maximum stroke of 60 micro meters. The results predicted by the algorithm closely resembled the experimental results and hence the authors concluded that they were successful in demonstrating the feasibility of their approach.

Robert M. Hall et al [9] inspects the stability of chinned aircrafts with different cross-sectional fuselage area. He calculated the pitching, yawing and rolling moment for these sections at different angles of attack and sideslip. Two fuselage sections of chine angles 30 and 100 degrees were subjected to winds in the upright and inverted configurations. 276 sensors placed around the body of the aircraft picked up the instantaneous pressure at different angles of attack. The data collected revealed that sharper chines resulted in larger lift coefficients and greater longitudinal stability. Upright configurations exhibited more stability than inverted ones.

Wen Jing et al [10] investigates the static directional stability of an aircraft at different angles of attack. The part of the aircraft contributing most to the instability was primarily identified and then probed into. A model of the aircraft consisting of 194 pressure taps was subjected to winds of $35 \mathrm{~m} / \mathrm{s}$ at angles of attack ranging from 0 to 46 degrees under the influence of sideslip. The vortices and their flow were extensively studied at the vertical tail and the fuselage body. The net effect of the yawing moment and windward leeward pressure difference verses stability at different attack- sideslip angles was recorded. It was noted that maximum instability was caused by the vertical tail and the fuselage body. The stability at the tail increased 
when the windward vortex completely broke down. Instability in the middle of the fuselage rapidly increased with an increase in angle of attack.

T.P. Ratvask et al [11] investigated the effects of icing on aircraft stability and control. For this purpose, a DH-6 Twin Otter aircraft was considered. The aircraft was tested in the normal as well as in iced conditions (Styrofoam attached to horizontal and vertical stabilizers). The aircraft was flown with 3 different thrust coefficients $(0.14,0.07$, 0.00 ), to determine the power effect (engine torque and power coefficient etc.). Maximum Likelihood algorithm was used to analyse the extensive data collected on flight manoeuvres. Modified Stepwise Regression method was then used to obtain stability. Static stability decreased by $10 \%$ due to tail ice. A drastic decrease was seen in directional stability during the zero-thrust case. Yaw damping and pitch damping remained unaffected.

Fabrizio Nicolosi et al [12] wrote a comprehensive analysis on the Tecnam P2006T a four-seater twin propeller aircraft during its flight. Flight quality was assessed, and stability derivatives were determined using longitudinal parameter estimation techniques. The above aircraft designed by Luigi Pascale was subjected to real time flight test, after its scaled model had been subjected to extensive testing in the wind tunnel. Static-dynamic lateral and longitudinal stability assessment of the aircraft was carried out by subjecting the aircraft to a number of flight maneuvers. Parameter estimation during flight was done using the Maximum Likelihood Method. The wind tunnel and full-scale test coincided with little discrepancy. The frequency response during the maneuvers fell well within the thumbprint criterion. The aircraft designed was successfully tested.

Fabrizio Nicolosi et al [13] tested a scaled model of the Tecnam P2012 in a wind tunnel and compared the values obtained to those obtained by CFD analysis. Estimating the lateral and longitudinal stability was the primary objective. The model fitted with strain gauge balances and was subjected to winds of $40 \mathrm{~m} / \mathrm{s}$. The effect of the flap on longitudinal stability and lift was extensively tested along with the effects of the horizontal tail. Lateral directional stability was also looked into. A CFD analysis looks into the wing span loading and drag breakdown along with the analysis of those mentioned above. The neutral point of the aircraft shifts by $3 \%$ with full flap deflection at zero angle of attack. It was also seen that the winglets and nacelles caused maximum drag. The pressure variations on the Tecnam P2012 are shown in figure 2. The Results obtained were used for the final design of the aircraft

Masoud Mirzaei et al [14] computed the drag due to the aft body of a cargo aircraft. A scaled modified model of the passenger jet was subjected to streams of air in a single return wind tunnel at angles of attack between -10 to 20 degrees. Numerical models of 4 modified aft created from an optimised prototype were subjected to 2 simulations (Spalart-Allmaras (SA) modelling and Large Eddy Simulation (LES): more computational effort). The LES model proved to be more accurate (as compared to the wind tunnel model) than the SA. The cargo vehicle saw considerable increase in drag coefficient (28\%) due to flow separation near the ramp door. Further optimisation led to the decrease in drag coefficient.

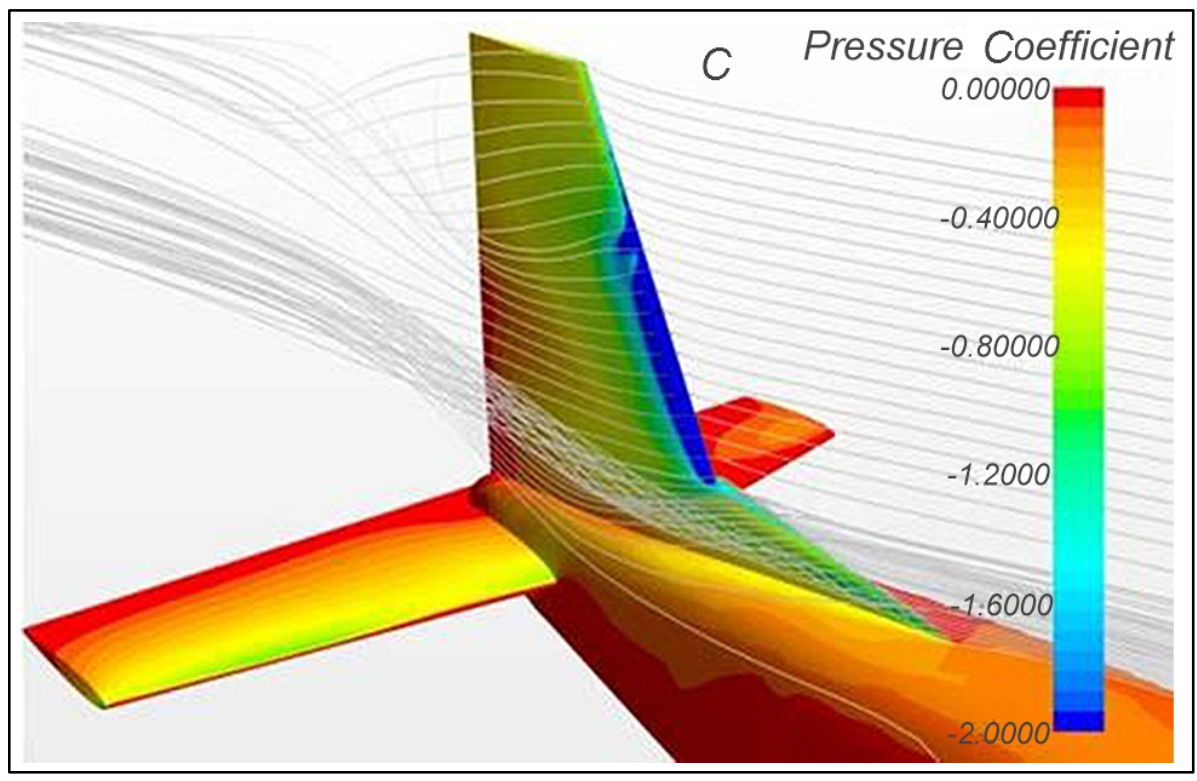

Figure 2. CFD analysis of the vortices on Tecnam P2012 [13] 
DENG Xueying et al [15] studied the flow of asymmetric vortices over a slender body at high angles of attack. Mechanisms of vortices were studied and its evolution due to perturbations on the nose was presented. The evolution of a vortex from a symmetric to an asymmetric state was looked into using slender bodies of different nose shapes. To prove that the non-determinacy of the asymmetric vortices was due to the non-determinacy of the perturbation, a model was subjected to two different wind stations. Side forces acting were being measured in order to correlate it with asymmetric vortex flow. Active control of forebody vortices was also looked into. The behaviour of the asymmetric vortices was controlled mainly due to the irregularities on the nose. The findings of this paper are many and can be widely used in fields of aerodynamic optimisation and design.

Shi Wei et al [16] analyses the behaviour of vortices over the chinned fuselage when subjected to different angles of attack. A scaled down model of the fuselage section is bombarded with winds of $35 \mathrm{~m} / \mathrm{s}$ at different angles of attack and side forces are measured. Artificial tip perturbations are introduced on the nose at different circumferential angles. Pressure taps measure the variation in pressure at 22 different circumferential nodes. With the variation in the angle of attack between 0 to 70 degrees the vortices morph from symmetric to asymmetric and finally they breakdown. The nature of the asymmetric vortices is determined. The vortices on the side of the distortion are higher than the ones on the opposite side. This results in a side force acting towards the lower vortex. A conclusive idea of the vortices acting on the windward and leeward side is established.

\section{Numerical Analysis of Aircraft Fuselage}

S. Dey et al [17] devised a set of experiments through which a comprehensive account of the behaviour of doubly curved composite shells when exposed to various time varying loads. The input parameters chosen were ply-orientation angle, radius of curvatures, material properties, dynamic and static loads etc within appropriate constraints. The moving least square method in tandem with original Monte carlo simulation method and finite element modelling were used to mathematically derive and validate the results. They concluded that a variability of the input parameters like aspect ratio, ply orientation angle and radius of curvature had an impact on the structural instability of the composite panels and should be a prevalent factor during the design and manufacturing process of the panels.

C. Suresh et al [18] delves into the history of aircrafts and describes the various attempts to increase the lift by utilizing bi or even tri wing concepts. But due to their high inefficiencies, a C-type mono-wing design was settled for.
The instrumental parameter to be considered during wing design is the lift to drag ratio, and the main aim of their work was to definitively prove that the C-wing design is considerably better with respect to a straight wing design with the same surface wing area across a wide range of operational flight speeds and angles of attack. The main objective is to conduct a comparative aerodynamic performance study along the parameters of drag, lift and stall using CFD analysis. The three-dimensional model of the wing designs was made using CATIA and ANSYS CFX softwares. The post processing results indicate a superior performance and lift to drag ratios over a wide range of angle of attacks for the non-planer $\mathrm{C}$-wing design over the plane wing design.

Cristofaro $M$ et al [19] attempted to generate an aerodynamic data which would form the baseline for the simulation of a reginal jet aircraft which could be used to check the performance and aerodynamic stability of the jet. Aerodynamic models were not only confined to CFD analysis, but also included semi-empirical approaches which utilized data from wind tunnel tests to validate the results generated from the simulations. A baseline geometry was first established from which the impacts of changes in the geometry of the aircraft could be compared to. They noticed that a change in the wing sweep angle had a bigger impact on the stability than the wing aspect ratio of the aircraft. An increase in the sweep angle led to an increase in the angle of attack which in turn led to a negative elevator deflection to trim the aircraft. They indicated that any future work would further improve on the dataset to optimally refine the aircraft geometry and demonstrate the ability of CFD analysis to design aircraft geometry.

YongxiLyu et al [20] attempted to construct and design a control law under unsteady aerodynamics. They utilized Extreme Learning Machine (ELM), and to build the algorithms, the authors made use of backstepping and daisy chain control allocation. The model around which the equations and simulations are conducted is an advanced fighter with blended wings, leading to edge flaps and trailing flaps which are distributed along the $\mathrm{X}$ axis of the body in a mirror image fashion. A wind tunnel test is conducted which is used as reference against which the designed control low is validated. The results indicate that The ELM model describing the unsteady aerodynamics showed promising fidelity to the data from the wind tunnel test. The authors concluded that the model had accomplished to accurately represent the aerodynamic forces experienced by the fighter jet under normal and unsteady conditions. They successfully show that a control law designed by combing Radial Basis Function (RBF) network and the Dynamic Surface Control (DSC) method is instrumental in solving unsteady aerodynamic problem statements.

Juhee Lee [21] performed a computational analysis with an optimized wing structure in an attempt to construct a 
three-dimensional WIG aircraft consisting of a fuselage and a wing with a reduced horizontal tail which is normally necessary to counteract the vibrations and offer stability but is detrimental to static stability when ground effects are not in play. They first constructed a mathematical model describing the Wing-In-Ground (WIG) model. This model, in two configurations, wings with and without ground effects, were validated using CFDc analysis followed by flow analysis which utilized three-dimensional Navier-Stokes equations. In total, six stability configurations were evaluated like, static height stability, varying locations for the center of gravity, varying degrees of trim for flight underground effects etc. From the results, they concluded that the optimised wings offer improved aerodynamic performance. They also deduced that to achieve higher stability, ideally the tail should be placed higher than that was considered in this study and to satisfy the stability requirements, a tail wing one-fourth the size of the main wing area was required.

The BWB Aircraft is more susceptible to turbulent airflows due to the lack of a vertical tail. Sami Ammar et al [22] therefore performed a stability analysis on a 200 passenger self-designed BWB and compares the results to an equivalent conventional A320 and B747 airbus. CEASIOM a designing platform was used for conceptualizing the BWB under parameters like drag and engine performance. CATIA was later used for the completely optimized modelling. Performance and stability of the above vehicles were analyzed under similar conditions. Performance analysis indicated reduction in take-off weight and distance with improved lift drag ratio for the BWB. Stability analysis indicated instability along the lateral axis of BWB during Dutch Roll because of low damping ratio.

Toshihiro Ikeda et al [23] explores the aerodynamics of a BWB Aircraft design. A new configuration for ferrying passengers was analysed and presented as a viable alternative to the traditional discrete fuselage wings structure. Certain parameters like load to drag ratio, weight of the BWB and the passenger capacity, were used to design (using CATIA V5, FLUENT 6.2 softwares) and optimise (CFD analysis) the vehicle. NASA's inventory of research on BWB was optimised and used to carter to the needs of the authors design. The optimised BWB created turbulent eddies, which couldn't be resolved. The load to drag ratio achieved by the BWB was 1.4 times greater than that of a regular aircraft. Noise emission of the BWB reduced due to smaller drag values and lesser engine thrust requirements. The BWB with its increasing advantages seems to be the vehicle for the future.

Moving Surafce Boundary-layer Control (MSBC) is a fringe idea which could be utilized to improve the aerodynamic performance of any wing owing to its ability to delay the separation of boundary layer of airflow from the surface of the aero foil. One configuration through which this is implemented is by attaching a rotating cylinder rotating counter to the direction of the airflow. Abdus Salam et al [24] utilizes this concept and envisages the design of a wing with circle at its leading edge and investigates the gain in aerodynamic performance under varying circumstances along a range of angles of attack. They chose to modify a NACA-0010 model airfoil so as to satisfy the requirements of this experiment and built a 3-D model of the design. The results from the computational analysis indicated an increase in the lift component of the airfoil by 13.75 percent at the detriment of increased drag due to increased friction. The authors remarked that the benefits offered substantiated measures to reduce the drag produced through structural modifications like tapers, winglets etc.

Flutter is an unfavorable consequence of unsteady aerodynamic and elastic forces acting on an aircraft during the act of flying, and in extreme conditions can lead to catastrophic failure. Y. J. Choia et al [25] tackled this phenomenon in T-tail among transport aircrafts by conducting an empennage flutter analysis under the event of a modification in the fuselage length due to plug-in segments. They devised an experiment where two models were constructed using FEM, one acting as a base test and the other model with 120 inches added to the fuselage length acting as the plug-in segment. They used MSC.NASTRAN to check parameters like stiffness, mass distribution and aerodynamics. They first conducted a normal modal analysis acting as the reference against which data would be cross verified. This was followed by the flutter analysis test. Upon going through the results, they concluded that the addition of the plug-in reduced the empennage flutter mode but was found to have an adverse effect on complex flutter mode for wing and the empennage section.

C. Edward Lan et al [26] used Fuzzy-Logic (FL) to understand the aerodynamic effect during landing. In the period of touchdown, air around the aircrafts collapses into a more complex unstable form which causes hard landings and runway veer-offs. The flight variables during descent were used to generate the possible stability and control derivatives which were used in computing the overall stability using FL inversion. The stability values generated, synchronised with those obtained from a Flight Data Recorder on a twin jet transport aircraft. It was noticed that roll controls while landing were very much different from those experienced during flight. The control over the rudder to avoid veer-off was decreased due to the turbulent air and strong cross winds. Air is noticed to behave very differently during landings.

Zhenlong Wu et al [27] explored the lateral stability of an aircraft under heavy rain. A DHC-6 Twin Otter aircraft was subjected to numerical simulation and aerodynamic coefficients during rain were obtained using Eulerian-Lagrangian method. Strip theory and linear fitting processing was used to procure static and dynamic derivatives. Controllability of the aircraft was tested by 
analyzing its response to unit step changes in the control surface. The numerically obtained values for lift and drag were in harmony with those obtained experimentally. Of the 13 coefficients considered for lateral stability only four showed significant changes during rain, contributing to most of the instability experienced. Heavy rainfall adversely affected recovery of stability during Dutch roll mode, also increasing the time for recovery. The potency of the rudder and the aileron also reduced. Heavy rain decreases stability and controllability thereby increasing the chances of flight failure.

IlhanTuzcu et al [28] analysed the stability of a flexible aircraft due to flight dynamics and that due to aero-elasticity. An aircraft was first modelled such that the wings, fuselage and empennage could be distinctly identified. Unified formulation (UF), Flight dynamics of the quasi-rigid aircraft $(\mathrm{QR})$, Aero-elasticity of flexible components (AF) and Aero-elasticity of restrained flexible aircraft (AR) were the four frameworks then used in studying the models stability. Divergence and flutter speed were obtained under UF. Greater speeds resulted in negative stiffness and divergence, as the AF revealed. Cause of flutter was aerodynamic forces shown in the AR model. An analysis on the business jet showed that the most accurate model for closed loop control was the Unrestrained Aircraft one.

J. Mieloszyk et al [29] demonstrate the proficiency of computational power and constrained optimization in the efficient designing of an aircraft. The main objective of the research was to improve and expand preexisting models encompassing the whole aircraft. They decided to apply the optimization loop to a boxwinged aircraft along the parameters of aerodynamics, structure and dynamic stability with parameter specific constraints. The results of the optimization algorithms point to a better lift and optimal air flow aerodynamically, a change in the geometry to attain structural stability and reduced oscillations over wide tested airspeeds and altitudes. The results of the pressure simulations are present in figure 3 . The authors concluded that they were successful in effectively running and implementing the results of the constrained optimization problems to create the most efficient design despite of its unconventional nature. Though the pilot raised concerns regarding the limitations of the aircrafts handling capabilities, they believe that these minor grievances would be ironed out with further iterations.

Viken N. Koukounain et al [30], devised a series of experiments to study the vibro-acoustic response resulting in the change in thickness of the fuselage panel skin. The main objective was to develop reproducible semi-empirical procedure to hypothesize the dynamic response with certainty when under the turbulent boundary layer (TBL) excitation and the subsequent noise within the fuselage structure brought upon by vibrating fuselage skin. This TBL condition occurs when the aircraft is cruising at high altitudes. The experiments conducted were in line with international standards set by aviation bodies so as to be compliant with regulations.

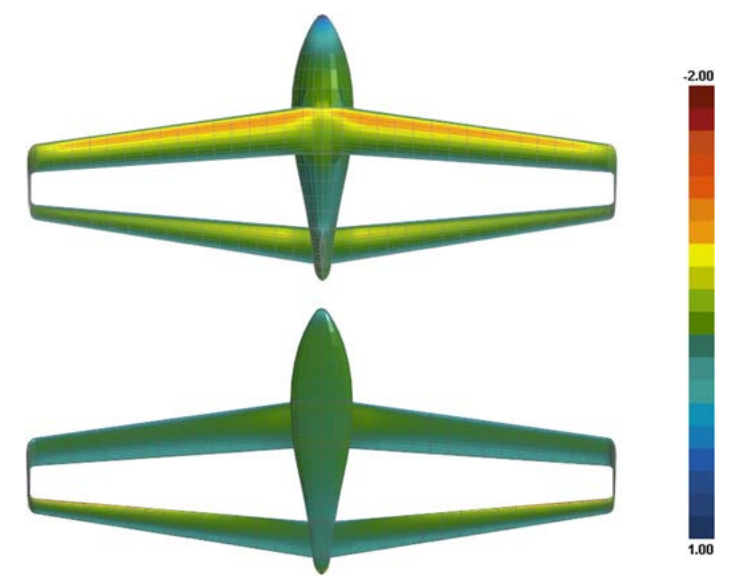

Figure 3. Pressure coefficient distribution for cruise conditions on the optimized aircraft. (Refer paper for further explanation regarding the colour gradient) [29]

From several parameters along which the phenomenon could be studied, sound pressure and transmission loss were chosen. Various external factors (dampening, flanking etc) affecting the efficacy of the data were taken into considerations in the algorithms and the final results were in line with experimental data. Hence the authors concluded that their approach was a low-cost way to better design fuselage structures in order to attenuate the vibrations generated due to various phenomena.

\section{Summary of Literature Review}

Permutation and combinations of many current theories and formulas have been pivotal in establishing new ground-breaking concepts in the recent past. Reshaping the fuselage into an elliptical structure proves more beneficial for the overall stability of the aircraft. Drag Reduction using new established algorithms proves more efficient. An optimisation algorithm was also generated for an aircraft in stormy weather conditions. The theoretical conceptualisation of new concepts has required a great amount of testing before finally being adapted into the aircraft. These theories are first validated by means of physical experiments or by the use of numerical software, before finally being implemented.

The success of every in- flight aircraft can be mapped back to the arduous hours dedicated by a delegation, tweaking the parameters of a wind tunnel. The indispensability of a wind tunnel is further established in this day and age, even with the presence of simulations involving Computational Fluid Dynamics (CFD). Stability under icing conditions is looked into and compiled from tests in the tunnels. Fuselage panels with all its composite variants are tested for structural stability. The introduction 
of perturbations to the nose of the aircraft and its effects on the vortices around the aircraft body are extensively tested

\section{Conclusions}

The relentless pursuit of improved performance has pushed the boundaries of aircraft design, forever changing the face of the aeronautics and aviation. The advancements made in subsidiary industries and sciences have demanded the fuselage to adapt with the times. This has seen a marked increase in the intensity of research conducted concerning unorthodox fuselage designs. This has inevitably led to challenges arising in the department of aerodynamics and stability. The field in general has resorted to unconventional solutions which require intense computational verification in tandem with real life experimentation. Some of the design philosophies tackled here are, boxwinged aircraft, BWD's, MSBC's etc. These concepts theoretically offer a more efficient solution to the problems faced by the aircraft industry today. The analyses of the stability of these fuselage designs were conducted on miniature models in wing tunnels and using simulation technologies. Great strides were also taken in further refining modern day wing and fuselage designs, subjecting them to a wide range of circumstances, ensuring their legality in line with aviation regulations. Various constructs to increase the stability were also addressed and required further experiments to be conducted to realise the true potential of these technologies.

Fuselage technologies are on the verge of experiencing revolutionary changes with ideas on the fringes finally taking center stage. These investments are bound to come to fruition with these aircrafts capable of transporting more with relatively lower fuel consumption and a smaller environmental footprint. All the technologies worked on in these papers require further experimentation and ideation for them to become more main-stream.

\section{REFERENCES}

[1] Yii-Mei Huang, Chun-Cheng Chen, Optimal design of dynamic absorbers on vibration and noise control of the fuselage, Computers \& Structures, Volume 76, Issue 6, Pages 691-702

[2] ParthaDey, M.K. Singha, Dynamic Stability Analysis Of Composite Skew Plates Subjected To Periodic In-Plane Load, Thin-Walled Structures, Volume 44, Pages 937-942

[3] Zhiquan Li, Pinqi Xia, Aeroelastic stability of full-span tiltrotor aircraft model in forward flight, Chinese Journal of Aeronautics, Volume 30, Issue 6, Pages 1885-1894

[4] Jesus Garicano-Mena, Estaban Ferrer, Silvia Sanvido, Eusebio Valero, A stability analysis of the compressible boundary layer flow over indented surfaces, Computers and
Fluids, Volume 160, Pages 14-25

[5] Liang QU, Yinghui LI, Haojun XU, Dengcheng ZHANG, Guoqiang YUAN, Aircraft Nonlinear Stability Analysis And Multidimensional Stability Region Estimation Under Icing Conditions, Chinese Journal of Aeronautics, Volume 30, Issue 3, Pages 976-982

[6] Wencheng Li, DongpingJin, Flutter suppression and stability analysis for a variable-span wing via morphing technology, Journal of Sound and Vibration, Volume 412, Pages 410-423

[7] N. Siepenkötter, W. Alles, Stability Analysis Of The Nonlinear Dynamics Of Flexible Aircraft, Aerospace Science and Technology, Volume 9, Pages 135-141

[8] C. Natale, G. Aurilio, G. De Maria, Modal Analysis And Vibration Control Of A Fuselage Aeronautical Panel, IFAC Proceedings Volumes, Volume 35, Issue 2, Pages 117-122

[9] Robert M. Hall, Impact of Fuselage Cross Section On The Stability Of A Generic Fighter, NASA Langley Research Center, AIAA-98-2725

[10] Wen Jing, Wang Yankui, Deng Xueying, An Experimental Investigation On Static Directional Stability, Chinese Journal of Aeronautics, Volume 29, Issue 6, Pages 15271540

[11] T.P. Ratvasky and R.J. Ranaudo, Icing Effects on Aircraft Stability and Control Determined from Flight Data, 31st Aerospace Sciences Meeting and Exhibit

[12] Fabrizio Nicolosi, Agostino De Marco, Pierluigi Della Vecchia, Stability, Flying Qualities And Longitudinal Parameter Estimation Of A Twin-Engine CS-23 Certified Light Aircraft, Aerospace Science and Technology, Volume 24, Pages 226-240

[13] FabrizioNicolosi, Salvatore Corcione, Pierluigi Della Vecchia, Commuter Aircraft Aerodynamic Design: Wind-Tunnel Tests And CFD Analysis, 29th International Congress Of The Aeronautical Sciences

[14] MasoudMirzaei, Mohammad HadiKarimi a, Mohammad Ali Vaziri, An Investigation of A Tactical Cargo Aircraft Aft Body Drag Reduction Based On CFD Analysis And Wind Tunnel Tests, Aerospace Science and Technology, Volume 23, Pages 263-269

[15] DENG Xueying, WANG Yankui, Asymmetric Vortices Flow Over Slender Body and Its Active Control at High Angle of Attack, The Chinese Society Of Theoretical And Applied Mechanics, Volume 20, Issue 6, Pages 567-579

[16] Shi Wei, Deng Xueying, Tian Wei, Wang Yankui, Influence of artificial tip perturbation on asymmetric vortices flow over a chined fuselage, Chinese Journal of Aeronautics, Volume 28, Issue 4, Pages 1016-1022

[17] S. Dey, T. Mukhopadhyay, S.K. Sahu, S. Adhikari, Stochastic dynamic stability analysis of composite curved panels subjected to non-uniform partial edge loading, European Journal of Mechanics And Solids, Volume 67, Pages 108-122

[18] C. Suresh, K. Ramesh, V. Paramaguru, Aerodynamic performance analysis of a non-planar C-wing using CFD, Aerospace Science and Technology, Volume 40, Pages 56-61 
[19] Cristofaro M., Wang Y., Da Ronch A., Towards Computational Flight Dynamics Of A Passenger Jet Aircraft, 29th Congress of the International Council of the Aeronautical Sciences, Pages 7-12

[20] YongxiLyu, Yuyan Cao, Weiguo Zhang, Jingping Shi, Xiaobo Qu, Dynamic surface control design of post-stall maneuver under unsteady aerodynamics, Aerospace Science and Technology, Volume 80, Pages 269-280

[21] Juhee Lee, Computational analysis of static height stability and aerodynamics of vehicles with a fuselage, wing and tail in ground effect, Ocean Engineering, Volume 168, Pages $12-22$

[22] Sami Ammar, Clement Legros, Jean-Yves Trepanier, Conceptual design, performance and stability analysis of a 200 passengers Blended Wing Body aircraft, Aerospace Science and Technology, Volume 71, Pages 325-336

[23] Toshihiro Ikeda, Cees Bil, Aerodynamic Performance of a Blendedwing-Body Configuration Aircraft, 25th International Congress of The Aeronautical Sciences

[24] Abdus Salam, Shuvrodeb Barman, Saifuddin Ahmed Atique, Asif ShahriarNafi, NafisaNawalProbha, Kh. Md. Faisal, CFD Approach to Analyze the Aerodynamics Potentiality of Rotary Leading Edge Aircraft Wing at Different Angle of Attack, Electrical Engineering and Information \& Communication Technology (ICEEICT), May 2015

[25] Y. J. Choi, S. W. Lee, W. Kang, S. K. Paek, Influence of Fuselage Plug-in on the Empennage Flutter Characteristics of a Transport Aircraft, 7th Asian-Pacific Conference on Aerospace Technology and Science, 7th APCATS

[26] C. Edward Lan, Ray C. Chang, Unsteady Aerodynamic Effects In Landing Operation Of Transport Aircraft And Controllability With Fuzzy-Logic Dynamic Inversion, Aerospace Science and Technology, Volume 78, Pages 354-363

[27] Zhenlong Wu, BenyinLv, Yihua Cao, Heavy Rain Effects On Aircraft Lateral/Directional Stability And Control Determined From Numerical Simulation Data, Aerospace Science and Technology, Volume 80, Pages 472-481

[28] IlhanTuzcu, On the stability of flexible aircraft, Aerospace Science and Technology, Volume 12, Pages 376-384

[29] J. Mieloszyk, T. Goetzendorf-Grabowski, Introduction of full flight dynamic stability constraints in aircraft multidisciplinary optimization, Aerospace Science and Technology, Volume 68, Pages 252-260

[30] Viken N. Koukounian, Chris K. Mechefske, Computational modelling and experimental verification of the vibro-acoustic behavior of aircraft fuselage sections, Applied Acoustics, Volume 132, Pages 8-18 